\title{
Generalized estimation of missing observations in nonlinear time series model using state space representation
}

\author{
Biwott K. Daniel ${ }^{1, *}$, Odongo O. Leo ${ }^{2}$ \\ ${ }^{1}$ Maseno University,Department of Statistics and Actuarial Science, Kenya \\ ${ }^{2}$ Kenyatta University,Department of Statistics, Kenya
}

Email address:

dbkiprotich@yahoo.com (B. K. Daniel)

\section{To cite this article:}

Biwott K. Daniel, Odongo O. Leo. Generalized Estimation of Missing Observations in Nonlinear Time Series Model Using State Space Representation, American Journal of Theoretical and Applied Statistics. Vol. 2, No. 2, 2013, pp. 21-28.

doi: $10.11648 /$ j.ajtas.20130202.13

\begin{abstract}
The aim of the study was to formulate a Time Series Model to be used in obtaining optimal estimates of missing observations. State space models and Kalman filter were used to handle irregularly spaced data. A non-Bayesian approach where the missing values were treated as fixed parameters. Simulated AR (1) data and corresponding estimated missing values were generated using a computer programme. Values were withheld and then estimated as though they were missing. The results revealed that simple exposition of state space representation for commonly used Time Series Models can be formulated.
\end{abstract}

Keywords: Model, Linear, Non-Linear, Simulated, Non-Bayesian

\section{Introduction}

One of the unfortunate facts facing data analysts is missing data. Data that are known to have been observed erroneously can be categorized as missing. Erroneous data can also wreak havoc with the estimation and forecasting of time series models.

In the past, estimation of missing observations has been considered among others by $[1,2,3,4,5,6]$. In particular, [7] and [8] developed state space method for dealing with missing observations in the long- memory context. More recently, [9] have given statistical analysis of incomplete long-range dependent data and the application of these procedures to the analysis of the annual minimum water levels of Nile River.

This paper addresses both theoretical and methodological issues related to the estimation of missing observations. Estimates are calculated by means of state space models and Kalman filter.

\section{State Space Models}

The linear state space system is given by, $\theta_{t+1}=\alpha_{t} \theta_{t}+$ $\beta_{t} \mu_{t+1}, y_{t}=A_{t-1} \theta_{t}+\beta_{t-1} v_{t}(2.1)$ where, $\theta_{t}$ and $\mu_{t}$ are $p \times 1$ vectors, $y_{t}$ and $v_{t}$ are $q \times 1$ vectors, $\alpha_{t}$ and $\beta_{t}$ are $p$ $\mathrm{x} \mathrm{p}$ matrices, and $A_{t}$ and $B_{t}$ are matrices of dimensions $p \times p$ and $q \times q$ respectively $\left\{y_{t}\right\}$ represents the observed time series, whereas $\alpha_{t}, A_{t}, \beta_{t}, B_{t}$ are known matrices of nonrandom function. The vectors $\left\{\mu_{t}\right\},\left\{v_{t}\right\}$ are independent each being a sequence of independent normal random vectors, having components with zero mean and unit variance. In order to handle various deviations which may occur in practice several generalization of (2.1) have been suggested.

In this paper we consider the model in (2.1) with random coefficients. We allow the coefficients in (2.1) to depend on past observations as follows;

$$
\alpha_{t}=\alpha\left(t, F_{t}^{y}\right), \beta_{t}=\beta\left(t, F_{t}^{y}\right), A_{t-1}=A\left(t-1, F_{t-1}^{y}\right)
$$
and $B_{t-1}=B\left(t-1, F_{t-1}^{y}\right)$, where $F_{t}^{y}$ denotes the $\sigma$-field generated by observationsup to time $t$.

We refer to (2.1) under this settings as the generalized model. This generalized model encompasses some of the non linear time series models that have been proposed in the literature.

(i) ARCH models: Supposed that $\alpha_{t}=\alpha, A_{t-1}=A$ and $B_{t} \cong 0$ so that,

$$
\theta_{t+1}=\alpha \theta_{t}+\beta_{t} \mu_{t+1}, y_{t}=A \theta_{t}
$$

This is the ARCH model described in [10] 
(ii) Dynamic linear state space models: When $\left\{\alpha_{t}\right\},\left\{\beta_{t}\right\}$ and $\left\{B_{t}\right\}$ are constant matrices and $A_{t-1}$ is a matrix of knownfunctions at $t-1$ (i.e. $A_{t-1}$ is $F_{t}^{y}$ measurable) the generalized model (2.1) becomes,

$$
\theta_{t}=\alpha \theta_{t}+\mu_{t}, y_{t}=A_{t-1} \theta_{t}+v_{t}
$$

Which is the state space model described in [11]

(iii) Doubly stochastic time series model (cf.Tj $\emptyset_{\text {stheim }}$ (1986)) [12]: When $\alpha_{t}=1, \beta_{t}=1 \mu_{t+1}=\varepsilon_{t+1}-$ $\varepsilon_{t-1}$ and $B_{t}=1$, then (2.1) becomes

$$
\theta_{t+1}=\theta_{t}+\mu_{t+1}, y_{t}=A_{t-1} \theta_{t}+v_{t} .
$$

This corresponds to the doubly stochastic time series model

$$
\theta_{t+1}=\theta_{t}+\varepsilon_{t}+\varepsilon_{t-1}, y_{t}=\theta_{t} f\left(t, F_{t-1}^{y}\right)+e_{t},
$$

considered in [13].

When $\left(t, F_{t-1}^{y}\right)=y_{t-1}$, this turns out to be a special case of the random Coefficient Autoregression (RCA) model of [14]. Moreover if we take, $\theta_{t}=\alpha_{t-1} \theta_{t-1}+\mu_{t}, y_{t}=\theta_{t}$ with $\alpha_{t-1}=\phi+\pi \exp \left(-\gamma y_{t-1}^{2}\right)$ then the generalized model (2.1) describes the exponential autoregressive model of [15].

Theorem 2.1 This theorem gives prediction and fixed point smoothing algorithms for the generalize model (2.1)

Let, $\hat{\theta}_{t}=E\left(\theta_{t} \backslash F_{t-1}^{y}\right), \Sigma_{\mathrm{t}}=\mathrm{E}\left[\left(\theta_{t}-\hat{\theta}_{t}\right)\left(\theta_{t}-\hat{\theta}_{t}\right)^{\mathrm{T}} \backslash\right.$ Ft-1y

Then,

$$
\begin{gathered}
\hat{\theta}_{t+1}=\alpha_{t} \hat{\theta}_{t}+k_{t}\left[y_{t}-\hat{y}_{t}\right] \\
\Sigma_{\mathrm{t}+1}=\beta_{t} \beta_{t}^{T}+\left(\begin{array}{c}
\left.\alpha_{t}-k_{t} A_{t-1}\right) \Sigma_{\mathrm{t}}\left(\alpha_{t}-k_{t} A_{t-1}\right)^{T}+ \\
k_{t} B_{t-1} B_{t-1}^{T} k_{t}^{T}
\end{array}\right.
\end{gathered}
$$

Where, $k_{t}=\alpha_{t} \Sigma_{\mathrm{t}} A_{t-1}^{T}\left[A_{t-1} \Sigma_{\mathrm{t}} A_{t-1}^{T}+B_{t-1} B_{t-1}^{T}\right]^{+}$and $\hat{y}_{t}=E\left(y_{t} \backslash F_{t-1}^{y}\right) ; M^{T}$ and $M^{+}$denote the transpose and the pseudo inverse respectively of a matrix $M$.

Proof of this theorem is a straight forward extension of results as presented by [16].

\section{Theorem 2.2}

Fort $>t_{j}$, let $\tilde{\theta}_{t_{j} \backslash t}=E\left(\theta_{t_{j}} \backslash F_{t}^{y}\right)$ be the estimate of $\theta_{t_{j}}$ base on the observations up to time t, $\tilde{\Sigma}_{\mathrm{t}}$ be the covariance matrix, $\tilde{\Sigma}_{t}=E\left[\left(\theta_{t_{j}}-\hat{\theta}_{t}\right)\left(\theta_{t_{j}}-\hat{\theta}_{t_{j}}\right)^{T} \backslash F_{t-1}^{y}\right] \operatorname{and} \Sigma_{\mathrm{t}}^{*}=$ $\mathrm{E}\left[\left(\theta_{t_{j}}-\hat{\theta}_{t_{j} \backslash t}\right)\left(\theta_{t_{j}}-\hat{\theta}_{t_{j} \backslash t}\right)^{T} \backslash F_{t-1}^{y}\right]$ Then, $\tilde{\theta}_{t_{j} \backslash t}=\tilde{\theta}_{t_{j} \backslash t-1}+\tilde{\mathrm{k}}_{t}\left(y_{t}-A_{t-1} \hat{\theta}_{t}\right), t>t_{j}$ Where $\tilde{\mathrm{k}}_{t}=\tilde{\Sigma}_{t} A_{t-1}^{T}\left[A_{t-1} \Sigma_{\mathrm{t}} A_{t-1}^{T}+B_{t-1} \mathrm{~B}_{\mathrm{t}-1}^{\mathrm{T}}\right]^{+}$,

$$
\begin{gathered}
\tilde{\Sigma}_{t+1}=\tilde{\Sigma}_{t}\left[\alpha_{t}-k_{t} A_{t-1}\right]^{T}, \Sigma_{t-1}^{*}=\Sigma_{\mathrm{t}} \text { for } t<t_{j} \text { and } \Sigma_{t}^{*}= \\
\Sigma_{t-1}^{*}-\widetilde{\Sigma}_{t} A_{t-1}^{T} \tilde{\mathrm{k}}_{t}^{T}, t \geq t_{j} .
\end{gathered}
$$

Proof of this theorem is a straight forward generalization of results in [16] or [17].

This research used state space models methodology which can handle irregularly spaced data. It also used the
Kalman filtering technique and a non-Bayesian approach where the missing values were treated as fixed parameters.

\section{Results and Discussions}

Autoregressive conditionally heteroscedastic (ARCH) type models and application to missing data

There are two different approaches of estimating missing values in time series;

1. A Bayesian approach:Which uses Kalman filtering technique.

2. A non-Bayesian approach: Where the missing values are treated as fixed parameters.

This paper uses the Kalman type recursive approach to estimate the missing values by replacing them with normal random variables. This type of approach may be viewed as one which uses a prior for the parameter which replaces the missing value.

\subsection{One Missing Observation}

Now we indicate an appropriate way to modify a given non linear time series to reflect the fact that the observation at time $\mathrm{m}$ is missing.Let $\left\{X_{t}\right\}$ be a time series in which $X_{m}$ is missing and $X_{n}^{\prime}=\left\{X_{1}, X_{2}, \ldots, X_{m+1}, \ldots, X_{n}\right\}$

If we know the first two conditional moments $E\left[X_{t+1} \backslash\right.$ $F t x$ and var $X t+1 \backslash F t x$, then $X t+1$ can be written as $X_{t+1}=E\left[X_{t+1} \backslash F_{t}^{x}\right]+X_{t+1}-E\left[X_{t+1} \backslash F_{t}^{x}\right](1)$

Suppose that the time series $X_{t}$ satisfies $E\left[X_{t+1} \backslash F_{t}^{x}\right]=$ $\alpha_{t-1} X_{t}$ and $X_{t+1}-E\left[X_{t+1} \backslash F_{t}^{x}\right]=\beta_{t-1} \mu_{t+1}$ (2)

Where $\alpha_{t-1}$ and $\beta_{t-1}$ are $F_{t-1}^{x}$ measurable and $\left\{\mu_{t}\right\}$ is an i.i.d. $\sim N(0,1)$ sequence. Then $X_{t+1}$ has ARCH representation.

$$
X_{t+1}=\alpha_{t-1} X_{t}+\beta_{t-1} \mu_{t+1}
$$

The restriction in (2) is introduced to apply the recursive approach. Now we consider the estimation of a missing observation as a parameter estimation problem in a particular formulation of the generalized model (2.1).

$$
\begin{gathered}
\theta_{t+1}=\alpha_{t-1} \theta_{t}+\beta_{t-1} \mu_{t+1}, \operatorname{Var}\left[X_{t+1} \backslash F_{t}^{x}\right](4) \\
y_{t}=A_{t-1} \theta_{t}+B_{t-1} v_{t} \text { with } A_{m-1}=0, B_{m-1}=1, A_{t}= \\
1, t \neq m-1 ; B_{t}=0, t \neq m-1 ;
\end{gathered}
$$

Then $Y=\left(X_{1}, X_{2}, \ldots, X_{m-1}, v_{m}, X_{m+1}, X_{m+2}, \ldots, X_{n}\right)$ is the extended observed series. Here $v_{m}$ is a random variable replacing the missing observation.

Such a formulation was also considered in Abraham and Thavaneswaran (1991) [1]

Using theorem 2.1 and 2.2 we have equations,

$k_{t}=\alpha_{t-1} \Sigma_{\mathrm{t}} A_{t-1}\left(A_{t-1}^{2} \Sigma_{\mathrm{t}}+B_{t-1}^{2}\right)^{+}$and $\Sigma_{\mathrm{t}+1}=\beta_{t-1}^{2}+$ $\left(\alpha_{t-1}-k_{t} A_{t-1}\right)^{2} \Sigma_{\mathrm{t}}+k_{t}^{2} B_{t-1}^{2}$.

Substituting the values of $A_{t}, A_{t-1}, B_{t}$ and $B_{t-1}$ gives

$$
\begin{gathered}
k_{t}=\alpha_{t-1} \Sigma_{\mathrm{t}}\left[\Sigma_{\mathrm{t}}\right]^{+} \\
k_{t}=\alpha_{t-1}
\end{gathered}
$$

For $t \neq m$ and 


$$
\Sigma_{\mathrm{t}+1}=\beta_{t-1}^{2}
$$

Alsofor $t=m, k_{m}=\alpha_{m-1} \Sigma_{\mathrm{m}} A_{m-1}\left(A^{2}{ }_{m-1} \Sigma_{\mathrm{m}}+\right.$ $B m-12+$,

But $A_{m-1}=0$, hence $k_{m}=0$ and $\Sigma_{\mathrm{m}+1}=\beta_{m-1}^{2}+$ $\left(\alpha_{m-1}-k_{m} A_{m-1}\right)^{2} \Sigma_{\mathrm{m}}+k_{m}^{2} B_{m-1}^{2}$ but $k_{m}=0$ to give $\Sigma_{\mathrm{m}+1}=\beta_{m-1}^{2}+\alpha_{m-1}^{2} \Sigma_{\mathrm{m}}(7)$

Where from (6) whent $=m$ we have $\Sigma_{\mathrm{m}+1}=\beta_{m-1}^{2}$ so that $\Sigma_{\mathrm{m}}=\beta_{m-2}^{2}$.

Substituting for $\Sigma_{\mathrm{m}}$ in (7) we have $\Sigma_{\mathrm{m}+1}=\beta_{m-1}^{2}+$ $\alpha_{m-1}^{2} \beta_{m-2}^{2}(8)$

Also from theorem 2.2 we have $\tilde{\Sigma}_{t-1}=\tilde{\Sigma}_{t}\left(\alpha_{m-1}-\right.$ $\mathrm{kmAm}-1$ but $\mathrm{km}=0$ so that

$$
\tilde{\Sigma}_{t-1}=0
$$

and $\tilde{\Sigma}_{m+1}=\tilde{\Sigma}_{m}\left(\alpha_{m-1}-k_{m} A_{m-1}\right)$. But $k_{m}=0$ so that $\tilde{\Sigma}_{m+1}=\tilde{\Sigma}_{m} \alpha_{m-1}^{2}$, also $\tilde{\Sigma}_{m}=\Sigma_{\mathrm{m}}=\beta_{m-2}^{2}$.

Hence, $\widetilde{\Sigma}_{m+1}=\alpha_{m-1}^{2} \beta_{m-2}^{2}(10)$

We also have from theorem (2.2) $\tilde{\theta}_{t_{j} \backslash t}=\tilde{\theta}_{t_{j} \backslash t-1}+$ $\tilde{\mathrm{k}}_{t}\left(y_{t}-A_{t-1} \hat{\theta}_{t}\right)$, but in this case $t=m$ and $A_{t}=1$ gives, $\tilde{\theta}_{m \backslash t}=\tilde{\theta}_{m \backslash t-1}+\tilde{\mathrm{k}}_{t}\left(y_{t}-A_{t-1} \hat{\theta}_{t}\right)$, and at $t=m+1$

$$
\tilde{\theta}_{m \backslash m+1}=\tilde{\theta}_{m \backslash m}+\tilde{\mathrm{k}}_{m+1}\left(y_{m+1}-\hat{\theta}_{m+1}\right)(11)
$$

Since, $\theta_{t}=y_{t}=X_{t}$. To find $\tilde{\theta}_{m \backslash_{m}}$ we have that $\tilde{\theta}_{m \backslash_{m}}=$ $E\left[\theta_{m} \backslash F_{m}^{y}\right]=E\left[\left(\alpha_{m-2} \theta_{m-1}+\beta_{m-2} \mu_{m}\right) \backslash F_{m}^{y}\right]$

$=\alpha_{m-2} \hat{\theta}_{m-1}=\alpha_{m-2} X_{m-1}$.

Next, $\hat{\theta}_{m+1}=E\left[\theta_{m+1} \backslash F_{m-1}^{y}\right]=E\left[\left(\alpha_{m-2} \theta_{m}+\right.\right.$ $\beta m-1 \mu m+1 \backslash F m y$ which gives $\theta m-1=\alpha m-1 \theta m$ and

$\hat{\theta}_{m}=\alpha_{m-2} \hat{\theta}_{m-1}=\alpha_{m-2} X_{m-1}$.Combining this gives $\hat{\theta}_{m+1}=\alpha_{m-1} \alpha_{m-2} X_{m-1}$.

Moreover, $\tilde{\mathrm{k}}_{t}=\widetilde{\Sigma}_{t} A_{t-1}^{T}\left[A_{t-1} \Sigma_{\mathrm{t}} A_{t-1}^{T}+B_{t-1} B_{t-1}^{T}\right]^{+}$

$$
\tilde{\mathrm{k}}_{t}=\frac{\widetilde{\Sigma}_{t}}{\Sigma_{\mathrm{t}}}
$$

Since $A_{t-1}=1, B_{t-1}=0$ and at $t=m+1(12)$ leads to

$$
\tilde{\mathrm{k}}_{m+1}=\frac{\widetilde{\Sigma}_{m+1}}{\Sigma_{\mathrm{m}+1}}=\frac{\alpha_{m-1} \beta_{m-2}^{2}}{\beta_{m-1}^{2}+\alpha_{m-1}^{2} \beta_{m-2}^{2}} \text { from (8) and (10). }
$$

Thus the estimate of the $m t h$ observation based on $X_{m-1}$ is $\tilde{X}_{m \backslash_{m+1}}=\alpha_{m-2} X_{m-1}+\frac{\alpha_{m-1} \beta_{m-2}^{2}}{\beta_{m-1}^{2}+\alpha_{m-1}^{2} \beta_{m-2}^{2}}\left[X_{m+1}-\right.$ $\alpha m-1 \alpha m-2 X m-1(14)$

This simplifies to

$$
\tilde{X}_{m \backslash m+1}=\frac{\alpha_{m-2} \beta_{m-1}^{2} X_{m-1}+\alpha_{m-1} \beta_{m-2}^{2} X_{m+1}}{\beta_{m-1}^{2}+\alpha_{m-1}^{2} \beta_{m-2}^{2}}
$$

Moreover, for a nonlinear model of the form

$X_{t+1}=\phi X_{t}+\mu_{t+1}$ in which the $m t h$ observation $X_{m}$ is missing, the estimate of $X_{m}$ based on $F_{m+1}^{y}$ is given by

$$
\tilde{X}_{m \backslash m+1}=\frac{\phi X_{m-1}}{1+\phi^{2} X_{m-1}^{2}}\left[X_{m-2}+X_{m+1}\right]
$$

Autoregressive models with deterministic time varying coefficient:

Model of the form

$$
X_{t}-\alpha(t, \phi) X_{t-1}=\mu_{t}
$$

have been found to be quite useful, in particular in signal processing [18] as in 3.15, it can be shown that the estimate $\tilde{X}_{m \backslash m+1}$ of the missing observation based on $F_{m+1}^{y}$ is given by;

$$
\tilde{X}_{m \backslash m+1}=\frac{\alpha(m+1, \phi) X_{m+1}+\alpha(m, \phi) X_{m-1}}{1+\alpha^{2}(m+1, \phi)}
$$

\section{Bilinear models:}

Consider the model $X-\phi X=c u_{t}+\beta X_{t-2} u_{t}$

The estimation of missing observation, $X_{m}$, can be obtained by writing the model as $X_{t}=\alpha_{t-2} X_{t-1}+\beta_{t-2} u_{t}$

Where $\alpha_{t-2}=\phi$ and $\beta_{t-2}=c+\beta X_{t-2}$. Hence the estimate of $X_{m}, \tilde{X}_{m \backslash m+1}=E\left[X_{m} \backslash F_{m+1}^{y}\right]$ and is given by

$$
\tilde{X}_{m \backslash m+1}=\frac{\phi \beta_{m-2}^{2} X_{m+1}+\phi \beta_{m-1}^{2} X_{m-1}}{\beta_{m-1}^{2}+\phi^{2 \beta_{m-2}^{2}}}
$$

\subsection{Two Consecutive Missing Observations}

(a) The estimate of $m \boldsymbol{t h}$ observation based on $X_{m+2}$.

We consider a slightly modified form of the model (3) in which we let $\beta_{t}^{2}=\sigma^{2}, X_{t+1}=\alpha_{t-2} X_{t-1}+\beta_{t-2} u_{t+1}$

Here $X_{m}$ and $X_{m+1}$ missing and $\alpha_{t}$ is $F_{t}^{x}$ measurable. The problem is to estimate $X_{m}$ based on the available data

$$
X_{n}^{\prime}=\left(X_{1}, X_{2}, \ldots, X_{m-1}, X_{m+2}, \ldots, X_{n}\right) .
$$

The corresponding state space model may be written as

$$
\left.\begin{array}{c}
\theta_{t+1}=\alpha_{t-1} \theta_{t}+\beta_{t-1} \mu_{t+1} \\
X_{t}=A_{t-1} \theta_{t} \\
y_{t}=A_{t-1} \theta_{t}+B_{t-1} v_{t}
\end{array}\right\}
$$

Where $A_{m-1}=A_{m}=0, B_{m-1}=B_{m}=1, A_{t}=1$, for $t \neq m ; B_{t}=0, t \neq m, m-1$

Then $Y=\left(X_{1}, X_{2}, \ldots, X_{m-1}, v_{m}, X_{m+1}, X_{m+2}, \ldots, X_{n}\right)$ is the extended observed series.

Here $v_{m}$ is a random variable replacing the missing observation. By theorem (2.1) and (2.2) it can be shown that for $t \neq m, m+1, k_{t}=\alpha_{t-2}$ and $k_{m}=k_{m+1}=k_{m+2}=0$.

Also from theorem 2.2 we have the following equations

$\tilde{k}_{t}=\tilde{\Sigma}_{t} A_{t-1}\left[A_{t-1}^{2}+\beta_{t-1}^{2}\right]^{+}, \tilde{\Sigma}_{t+1}=\tilde{\Sigma}_{t}\left(\alpha_{t-1}-k_{t} A_{t-1}\right)$.

Hence we have $\tilde{k}_{t}=\widetilde{\Sigma}_{t}\left[\tilde{\Sigma}_{t}\right]^{+}=\frac{\widetilde{\Sigma}_{t}}{\Sigma_{t}}=1$ (17)

which gives $\tilde{\Sigma}_{t+1}=\tilde{\Sigma}_{t} \alpha_{t-1}(18)$

and because $A_{m}=A_{m-1}=0 ; B_{m}=B_{m-1}=1 ; A_{t}=$ $1, B_{t}=0$ at $t \neq m, m+1$ we also get

$$
\tilde{k}_{m}=\tilde{k}_{m+1}=0
$$

It can be shown that $\tilde{\Sigma}_{m+2}=\alpha_{m-1} \alpha_{m-2} \beta_{m-3}^{2}$ (20)

We have from theorem (2.2) we have the following equation $\tilde{\theta}_{m \mid t}=\tilde{\theta}_{m \mid t-1}+\tilde{k}_{t}\left(y_{t}-A_{t-1} \hat{\theta}_{t}\right)$,

Which by taking expectation and simplifying gives

$$
\begin{gathered}
\hat{\theta}_{m+2}=\alpha_{m-1} \hat{\theta}_{m+1}, \hat{\theta}_{m+1}=\alpha_{m-2} \hat{\theta}_{m} \text { and } \hat{\theta}_{m}= \\
\alpha_{m-3} \hat{\theta}_{m-1}=\alpha_{m-3} X_{m-1}
\end{gathered}
$$


which combines to give

$$
\hat{\theta}_{m+2}=\alpha_{m-1} \alpha_{m-2} \alpha_{m-3} X_{m-1} .
$$

Thus the estimate of $m t h$ observation based on $X_{m+2}$ is

$$
\begin{array}{r}
\tilde{X}_{m \mid m+2}= \\
\alpha_{m-3} X_{m-1}+\tilde{k}_{m+2}\left(X_{m+2}-\alpha_{m-1} \alpha_{m-2} \alpha_{m-3} X_{m-1}\right)(21)
\end{array}
$$

But $\tilde{k}_{m+2}=\frac{\alpha_{m-1} \alpha_{m-2} \beta_{m-3}^{2}}{\beta_{m-1}^{2} \alpha_{m-1}^{2} \beta_{m-2}^{2}+\alpha_{m-1}^{2} \alpha_{m-2}^{2} \beta_{m-3}^{2}}$

Hence (21)becomes, $\tilde{X}_{m \mid m+2}=\alpha_{m-3} X_{m-1}+$

$$
\begin{aligned}
& \frac{\alpha_{m-1} \alpha_{m-2} \beta_{m-3}^{2}}{\beta_{m-1}^{2} \alpha_{m-1}^{2} \beta_{m-2}^{2}+\alpha_{m-1}^{2} \alpha_{m-2}^{2} \beta_{m-3}^{2}}\left(X_{m+2}-\right. \\
& \alpha m-1 \alpha m-2 \alpha m-3 X m-1,
\end{aligned}
$$

Which simplifies to give $\tilde{X}_{m \mid m+2}=$ $\frac{\alpha_{m-3}\left(\beta_{m-1}^{2} \alpha_{m-1}^{2} \beta_{m-2}^{2}\right) X_{m-1} \alpha_{m-1}^{2} \alpha_{m-2}^{2} \beta_{m-3}^{2} X_{m+2}}{\beta_{m-1}^{2} \alpha_{m-1}^{2} \beta_{m-2}^{2}+\alpha_{m-1}^{2} \alpha_{m-2}^{2} \beta_{m-3}^{2}}(23)$

Which is the required estimate of $m t h$ observation based on $X_{m+2}$.

(b) The estimate of the $(m+1)$ thobservation based on $\boldsymbol{X}_{\boldsymbol{m}+2}$.

We estimate $X_{m+1}$ observation based on available data $X_{n}^{\prime}=\left(X_{1}, X_{2}, \ldots, X_{m-1}, \hat{X}_{m}, X_{m+2}, \ldots, X_{n}\right)$, where $\hat{X}_{m}$ is the estimate of $X_{m}$ from the previous steps.

The corresponding state space models becomes,

$$
\left.\begin{array}{c}
\theta_{t+1}=\alpha_{t-4} \theta_{t}+\beta_{t-4} u_{t+1} \\
X_{t}=A_{t-1} \theta_{t} \\
y_{t}=A_{t-1} \theta_{t}+B_{t-1} v_{t}
\end{array}\right\}
$$

In this case we have $A_{m}=0, B_{m}=1$; and $B_{t}=0, A_{t}=$ 1 for $t \neq m+1$. The extended data will be, $Y=$ $\left(X_{1}, X_{2}, \ldots, X_{m-1}, \hat{X}_{m}, v_{m+1}, X_{m+2}, \ldots, X_{n}\right)$ where $v_{m+1}$ a normal random variable is replacing the missing observation. This estimate is now treated as if only one observation is missing in the data.

Using theorems 2.1 we have the modified form as,

$\Sigma_{t+1}=\beta_{t-3}^{2}+\left(\alpha_{t-3}-k_{t} A_{t-1}\right)^{2} \Sigma_{t}+k_{t}^{2} B_{t-1}^{2}$ and $k_{t}=$ $\alpha_{t-3} \Sigma_{t} A_{t-1}\left[A_{t-1}^{2} \Sigma_{t}-B_{t-1}^{2}\right]^{+}$, then

$k_{t}=\alpha_{t-3} \Sigma_{t}\left[\Sigma_{t}\right]^{+}=\alpha_{t-3}(25)$

and $\Sigma_{t+1}=B_{t-3}^{2}+\left(\alpha_{t-3}-k_{t}\right)^{2} \Sigma_{t}$

But from (25) $k_{t}=\alpha_{t-3}$, hence $\Sigma_{t+1}=B_{t-3}^{2}(26)$

Also from theorem (2.2) we have

$\tilde{k}_{t}=\tilde{\Sigma}_{t} A_{t-1}\left[A_{t-1}^{2} \Sigma_{t}-B_{t-1}^{2}\right]^{+}$and $\tilde{\Sigma}_{t+1}=\tilde{\Sigma}_{t}\left(\alpha_{t-3}-\right.$ ktAt-1T. Since, $A t=1, B t=0 ; t \neq m+1$ then this becomes $\tilde{k}_{t}=\Sigma_{t}\left[\Sigma_{t}\right]^{+}=\frac{\widetilde{\Sigma}_{t}}{\Sigma_{t}}$ as in (17) and $\tilde{\Sigma}_{t+1}=0(27)$

Since $A_{t-1}=1$ and $k_{t}=\alpha_{t-3}$. (28)

Setting $t=m+1 A_{m}=0, B_{m}=1$ we obtain $k_{m+1}=0$

and $\Sigma_{m+1}=\beta_{t-2}^{2}+\alpha_{m-2}^{2} \Sigma_{t+1}$. But from(26) at $t=m$, $\Sigma_{m+2}=\beta_{m-3}^{2}$

Hence,

$\Sigma_{m+2}=B_{m-2}^{2}+\alpha_{m-2}^{2} \beta_{m-3}^{2}(29)$

and $\tilde{\Sigma}_{m+2}=\tilde{\Sigma}_{m+2}\left(\alpha_{m-2}-k_{m+1} A_{m}\right)$ at $A_{m}=$

$0, k_{m+1}=0$, we have;

$\tilde{\Sigma}_{m+2}=\alpha_{m-2} \tilde{\Sigma}_{m+1}$ and $\tilde{k}_{m+1}=0$. Also, $\tilde{\Sigma}_{m+2}=$ $\alpha_{m-2} \tilde{\Sigma}_{m+1}=\alpha_{m-2} \Sigma_{m+1}=\alpha_{m-2} \beta_{m-3}^{2}$
$\tilde{\Sigma}_{m+2}=\alpha_{m-2} \beta_{m-3}^{2}(30)$

But at $\geq t_{j}, \tilde{\theta}_{t j \mid t}=\tilde{\theta}_{t_{j \mid t-1}}+\tilde{k}_{t}\left(y_{t}-\hat{\theta}_{t}\right)$ since $A_{t}=1$.

In this case if we let $t_{j}=m+1$ and $t=m+2$ we

have $\tilde{\theta}_{m+1 \mid m+2}=\tilde{\theta}_{m+1 \mid m+1}+\tilde{k}_{m+2}\left(y_{m+2}-\hat{\theta}_{m+2}\right)$

Where, $\tilde{\theta}_{m+1 \mid m+1}=E\left[\theta_{m+1} \mid F_{m+1}^{y}\right]=E\left[\left(\alpha_{m-3} \theta_{m}+\right.\right.$

$\beta m-3 u m+1 \mid F m+1 y$

Which gives $\tilde{\theta}_{m+1 \mid m+1}=\alpha_{m-3} \hat{X}_{m}$.

$\operatorname{Next} \hat{\theta}_{m+2}=E\left[\theta_{m+2} \mid F_{m+1}^{y}\right]=E\left[\left(\alpha_{m-2} \theta_{m+1}+\right.\right.$ $\beta m-2 u m+2|F m+1 y=\alpha m-2 E \theta m+1| F m+2 y=\alpha m-2 \theta$ $m$ thid $\hat{\theta}_{m+1}=\alpha_{m-2} \hat{\theta}_{m}$ which combines to give $\hat{\theta}_{m+2}=$ $\alpha_{m-2} \alpha_{m-3} \hat{\theta}_{m}=\alpha_{m-2} \alpha_{m-3} \alpha_{m-4} \hat{X}_{m}, \hat{\theta}_{m}=\hat{X}_{m}$ since the data has been observed up to time $t=m$.

Hence,

$$
\begin{aligned}
& \hat{X}_{m+1 \mid m+2}=\alpha_{m-3} \hat{X}_{m}+\tilde{k}_{m+2}\left(X_{m+2}-\alpha_{m-2} \alpha_{m-3} \hat{X}_{m}\right) \\
& \text { But, } \tilde{k}_{m+2}=\frac{\widetilde{\Sigma}_{m+2}}{\Sigma_{m+2}}=\frac{\alpha_{m-2} \beta_{m-3}^{2}}{\beta_{m-2}^{2}+\alpha_{m-2}^{2} \beta_{m-3}^{2}}(32) \\
& \text { Replacing for } \tilde{k}_{m+2} \text { in (31) we obtain } \tilde{X}_{m+1 \mid m+2}=
\end{aligned}
$$
$\alpha_{m-3} \hat{X}_{m}+\frac{\alpha_{m-2} \beta_{m-3}^{2}}{\beta_{m-2}^{2}+\alpha_{m-2}^{2} \beta_{m-3}^{2}}\left(X_{m+2}-\alpha_{m-2} \alpha_{m-3} \hat{X}_{m}\right)$.

Simplifying this

gives $\tilde{X}_{m+1 \mid m+2}=\frac{\alpha_{m-3}\left(\beta_{m-2}^{2}\right) \hat{X}_{m}+\alpha_{m-2} \beta_{m-3}^{2} X_{m+2}}{\beta_{m-2}^{2}+\alpha_{m-2}^{2} \beta_{m-3}^{2}}$

\subsection{Three Consecutive Missing Observations}

a) The estimate of $m t h$ observation based on $X_{m+3}$. The modified state space model is given by

$$
\left.\begin{array}{c}
\theta_{t+1}=\alpha_{t-3} \theta_{t}+\beta_{t-3} u_{t+1} \\
X_{t}=A_{t-1} \theta_{t} \\
y_{t}=A_{t-1} \theta_{t}+B_{t-1} v_{t}
\end{array}\right\}
$$

In this case we have three consecutive missing observations $X_{m}, X_{m+1}$ and $X_{m+2}$ for which we need to estimate $X_{m}$ before we estimate the remaining two observations respectively. The initial observation set is

$X_{n}^{\prime}=\left(X_{1}, X_{2}, \ldots, X_{m-1}, X_{m+3}, \ldots, X_{n}\right)$. The extended observed series is

$Y=\left(X_{1}, X_{2}, \ldots, X_{m-1}, v_{m}, X_{m+3}, \ldots, X_{n)}\right.$ where $v_{m}$ is a normal random variable replacing the missing observation.

Usingtheorems 2.1 and 2.2 we have the modified set of equations

$$
k_{t}=\alpha_{t-3} \Sigma_{t} A_{t-1}\left[A_{t-1}^{2} \Sigma_{t} \beta_{t-1}^{2}\right]^{+}
$$

and $\Sigma_{\mathrm{t}+1}=\beta_{t-3}^{2}+\left(\alpha_{t-3}-k_{t} A_{t-1}\right)^{2} \Sigma_{t}+k_{t}^{2} B_{t-1}^{2}(44)$

In this case $A_{m+1}=A_{m}=A_{m+1}=0, B_{m-1}=B_{m}=$ $B_{m+1}=1 ; A_{t}=1, B_{t}=0 ; t \neq m, m+1, m+2$.

This implies that, $k_{t}=\alpha_{t-3}$ and $\Sigma_{\mathrm{t}+1}=\beta_{t-3}^{2}$.

It can be shown that $\Sigma_{m+3}=\beta_{m-1}^{2} \alpha_{m-1}^{2} \beta_{m-2}^{2}+$ $\alpha_{m-1}^{2} \alpha_{m-2}^{2} \beta_{m-3}+\alpha_{m-1}^{2} \alpha_{m-2}^{2} \alpha_{m-3}^{2} \beta_{m-4}^{2}(35)$

and $\widetilde{\Sigma}_{m+3}=\alpha_{m-1} \alpha_{m-2} \alpha_{m-3} \beta_{m-4}^{2}$

We have $\widetilde{\theta}_{m \backslash_{m+3}}=\tilde{\theta}_{m \backslash_{m+2}}+\tilde{\mathrm{k}}_{m+3}\left(y_{m+3}-\hat{\theta}_{m+3}\right)$

$$
\tilde{k}_{m+3}=\frac{\widetilde{\Sigma}_{m+3}}{\Sigma_{m+3}}=
$$

$\frac{\alpha_{m-1} \alpha_{m-2} \alpha_{m-3} \beta_{m-4}^{2}}{\beta_{m-1}^{2}+\alpha_{m-1}^{2} \beta_{m-2}^{2}+\alpha_{m-1}^{2} \alpha_{m-2}^{2} \beta_{m-3}^{2}+\alpha_{m-1}^{2} \alpha_{m-2}^{2} \alpha_{m-3}^{2} \beta_{m-4}^{2}}$ 
Making successive substitution in (36) we get

$$
\begin{gathered}
\tilde{X}_{m \mid m+3}= \\
\alpha_{m-4} X_{m-1}+ \\
\alpha m-1 \alpha m-2 \alpha m-3
\end{gathered}
$$

$\beta m-42 \beta m-12+\alpha m-12 \beta m-22+\alpha m-12 \alpha m-22 \beta m-$ $32+\alpha m-12 \alpha m-22 \alpha m-32 \beta m-42 X m+3-\alpha m-1 \alpha m-$ $2 \alpha m-3 \alpha m-4 X m-1$.

Simplifying this gives

$\tilde{X}_{m \mid m+3}=$ $\alpha m-4 \beta m-12+\alpha m-12 \beta m-22+\alpha m-12 \alpha m-22 \beta m-3$ $2 X m-1+\alpha m-12 \alpha m-22 \alpha m-32 \beta m-42 X m+3 \beta m-12$ $+\alpha m-12 \beta m-22+\alpha m-12 \alpha m-22 \beta m-32+\alpha m-12 \alpha m$

$-22 \alpha m-32 \beta m-42(38)$

(b) The estimate of $(m+1)$ th observation based on

$\boldsymbol{X}_{m+3}$

This is treated as two missing observations as seen earlier the modified state space representations are

$$
\left.\begin{array}{c}
\theta_{t+1}=\alpha_{t-4} \theta_{t}+\beta_{t-4} u_{t+1} \\
X_{t}=A_{t-1} \theta_{t} \\
y_{t}=A_{t-1} \theta_{t}+B_{t-1} v_{t}
\end{array}\right\}
$$

The initial observation is

$X_{N}^{\prime}=\left(X_{1}, X_{2}, \ldots, X_{m-1}, \hat{X}_{m}, X_{m+3}, \ldots, X_{n}\right)$, wesee that $X_{m+1}$ and $X_{m+2}$ are missing hence, we need to estimate $X_{m+1}$. Then the extended observed series is $Y=$ $\left(X_{1}, X_{2}, \ldots, X_{m-1}, \hat{X}_{m}, v_{m+1}, X_{m+3}, \ldots, X_{n}\right)$, here $\hat{X}_{m}$ is estimate of $X_{m}$ baseon $X_{m+3}$ and $v_{m+1}$ is a normalrandom variable replacing the missing observation.

Using theorem 2.1 and 2.2 as before we have.

$A_{m}=A_{m+1}=0, B_{m}=B_{m+1}=1 ; A_{t}=1 ; B_{t}=0$;

$t \neq m+1, m+2, k_{t}=\alpha_{t-4}$ and

$\Sigma_{t+1}=\beta_{t-4}^{2}(40)$

Also $k_{m+1}=k_{m+2}=0$.

Next we have $\Sigma_{m+2}=\beta_{m-2}^{2}+\alpha_{m-3}^{2} \Sigma_{m+1}$ and $\Sigma_{m+3}=$ $\beta_{m-2}^{2}+\alpha_{m-2}^{2} \Sigma_{m+2}$

Hence $\Sigma_{m+2}=\beta_{m-2}^{2}+\alpha_{m-2}^{2} \beta_{m-3}^{2}+$ $\alpha_{m-2}^{2} \alpha_{m-3}^{2} \Sigma_{m+1}(41)$

From (40) at $t=m, \Sigma_{m+1}=\beta_{m-4}^{2}$, hence(41) becomes

$$
\Sigma_{m+3}=\beta_{m-2}^{2}+\alpha_{m-2}^{2} \beta_{m-3}^{2}+\alpha_{m-2}^{2} \alpha_{m-3}^{2} \beta_{m-4}^{2}
$$

From theorem 2.2 we have

$\widetilde{k_{t}}=\frac{\widetilde{\Sigma_{t}}}{\Sigma_{t}}$ and $\widetilde{\Sigma}_{t+1}=0$. Also $\widetilde{\Sigma}_{t+2}=\alpha_{m-3} \widetilde{\Sigma}_{t+1}$ and $\widetilde{\Sigma}_{t+3}=$ $\alpha_{m-2} \tilde{\Sigma}_{t+2}$

So that, $\tilde{\Sigma}_{t+3}=\alpha_{m-2} \alpha_{m-3} \tilde{\Sigma}_{t+1}=\alpha_{m-2} \alpha_{m-3} \Sigma_{m+1}$, where $\Sigma_{m+1}=\beta_{m-4}^{2}$.

Hence, $\tilde{\Sigma}_{t+3}=\alpha_{m-2} \alpha_{m-3} \beta_{m-4}^{2}(43)$

Also, $\widetilde{k_{t}}=\frac{\widetilde{\Sigma_{t}}}{\Sigma_{t}}$ so that, $\tilde{k}_{m+3}=\frac{\widetilde{\Sigma}_{m+3}}{\Sigma_{m+3}}$

Where from equations (42) and (43) we get $\tilde{k}_{m+3}=$

$$
\begin{aligned}
& \frac{\alpha_{m-2} \alpha_{m-3} \beta_{m-4}^{2}}{\beta_{m-2}^{2}+\alpha_{m-2}^{2} \beta_{m-3}^{2}+\alpha_{m-2}^{2} \alpha_{m-3}^{2} \beta_{m-4}^{2}}(44) \\
& \quad \text { Then } \widetilde{\theta}_{t_{j} \mid t}=\tilde{\theta}_{t_{\mid t-1}}+\widetilde{k}_{t}\left(y_{t}-A_{t-1} \hat{\theta}_{t}\right), t>t_{j}
\end{aligned}
$$

But $A_{t}=1$ and $t_{j}=m+1$ and $t=m+3$ so that,

$$
\tilde{\theta}_{m+1 \mid m+3}=\tilde{\theta}_{m+1 \mid m+2}+\tilde{k}_{m+3}\left(y_{m+3}-\hat{\theta}_{m+1}\right)(46)
$$

We have $\tilde{\theta}_{m+1 \mid m+2}=E\left[\theta_{m+1} \mid F_{m+2}^{y}\right]=E\left[\left(\alpha_{m-4} \theta_{m}+\right.\right.$ $\left.\left.\beta_{m-4} \mu_{m+1}\right) \mid F_{m+2}^{y}\right]=\alpha_{m-4} E\left(\theta_{m} \mid F_{m+2}^{y}\right)=\alpha_{m-4} \hat{\theta}_{m}$,

But $\hat{\theta}_{m}=\hat{X}_{m}$ since we have made an observation up to time $t=m$ hence, $\tilde{\theta}_{m+1 \mid m+2}=\alpha_{m-4} \hat{X}_{m}$.

Next, $\hat{\theta}_{m+3}=E\left[\theta_{m+3} \mid F_{m+2}^{y}\right]=E\left[\left(\alpha_{m-2} \theta_{m+2}+\right.\right.$ $\beta m-2 u m+3 \mid F m+2 y=\alpha m-2 \theta m+2$

$\theta_{m+2}=\alpha_{m-3} \hat{\theta}_{m+1}, \hat{\theta}_{m+1}=\alpha_{m-4} \hat{\theta}_{m}=\alpha_{m-4} \hat{X}_{m}$ and these combines to give

$\hat{\theta}_{m+3}=\alpha_{m-2} \alpha_{m-3} \hat{\theta}_{m+1}=\alpha_{m-2} \alpha_{m-3} \alpha_{m-4} \hat{\theta}_{m}$, which gives

$\hat{\theta}_{m+3}=\alpha_{m-2} \alpha_{m-3} \alpha_{m-4} \hat{X}_{m}$ (47)

Where $\hat{X}_{m}$ is the estimate of $X_{m}$ obtained ealier in the same section. Hence (46) becomes,

$$
\begin{aligned}
& \hat{X}_{m+1 \mid m+3}=\alpha_{m-4} \hat{X}_{m} \\
& +\tilde{k}_{m+3}\left(X_{m+3}-\alpha_{m-2} \alpha_{m-3} \alpha_{m-4} \hat{X}_{m}\right)
\end{aligned}
$$

$=\alpha_{m-4} \hat{X}_{m}$
$+\frac{\alpha_{m-2} \alpha_{m-3} \beta_{m-4}^{2}}{\beta_{m-2}^{2}+\alpha_{m-2}^{2} \beta_{m-3}^{2}+\alpha_{m-2}^{2} \alpha_{m-3}^{2} \beta_{m-4}^{2}}\left(X_{m+3}\right.$
$\left.-\alpha_{m-2} \alpha_{m-3} \alpha_{m-4} \hat{X}_{m}\right)$

Simplifying this gives

$$
\hat{X}_{m+1 \mid m+3}=\frac{\alpha_{m-4}\left(\beta_{m-2}^{2}+\alpha_{m-2}^{2} \beta_{m-3}^{2}\right) \hat{X}_{m}+\alpha_{m-2}^{2} \alpha_{m-3}^{2} \beta_{m-4}^{2} X_{m+3}}{\beta_{m-2}^{2}+\alpha_{m-2}^{2} \beta_{m-3}^{2}+\alpha_{m-2}^{2} \alpha_{m-3}^{2} \beta_{m-4}^{2}}
$$

\section{c) The estimate of $(m+2)$ th observation based on}

$X_{m+3}$.

This is treated as one missing observation since $X_{m}$ and $X_{m+1}$ has been estimated. Then we have the new set of observations as

$X_{N}^{\prime}=\left(X_{1}, X_{2}, \ldots, X_{m-1}, \hat{X}_{m}, \hat{X}_{m+1}, X_{m+3}, \ldots, X_{n}\right)$ the new state space representations are;

$$
\left.\begin{array}{c}
\theta_{t+1}=\alpha_{t-5} \theta_{t}+\beta_{t-5} u_{t+1} \\
X_{t}=A_{t-1} \theta_{t} \\
y_{t}=A_{t-1} \theta_{t}+B_{t-1} v_{t}
\end{array}\right\}
$$

Then

$X_{N}^{\prime}=\left(X_{1}, X_{2}, \ldots, X_{m-1}, \hat{X}_{m}, \hat{X}_{m+1}, v_{m+2}, X_{m+3}, \ldots, X_{n}\right)$ is the extended observed series. Here $\hat{X}_{m}$ and $\hat{X}_{m+1}$ are the estimates of $X_{m}$ and $X_{m+1}$ from the previous steps. $v_{m+2}$ is a normal random variable replacingthe missing observation. In this case $A_{m+2}=0, B_{m+2}=1 ; A_{t}=1, B_{t}=0 ; t \neq m+$ 2. Again from the theorems 2.1 and 2.2 we have

$$
\begin{array}{r}
k_{t}=\alpha_{t-5}, \Sigma_{t+1}=\beta_{t-5}^{2} \\
k_{m+2}=0, \Sigma_{\mathrm{m}+3}=\beta_{m-3}^{2}+\alpha_{m-3}^{2} \Sigma_{\mathrm{m}+2}
\end{array}
$$

Setting $t=\min (49)$ we obtain $\Sigma_{t+1}=\beta_{m-5}^{2}$ and $\Sigma_{t+2}=$ $\beta_{m-4}^{2}$.

Hence, (50) now becomes $\Sigma_{\mathrm{m}+3}=\beta_{m-3}^{2}+$ 


$$
\begin{aligned}
& \alpha_{m-3}^{2} \beta_{m-4}^{2}(51) \\
& \text { Also } \widetilde{k}_{t}=\frac{\widetilde{\Sigma}_{t}}{\Sigma_{t}} \text { So that, } \tilde{k}_{m+3}=\frac{\widetilde{\Sigma}_{m+3}}{\Sigma_{m+3}}(52) \\
& \quad \tilde{k}_{m+2}=0, \widetilde{\Sigma}_{m+3}=\alpha_{m-3} \widetilde{\Sigma}_{m+2}=\alpha_{m-3} \Sigma_{m+2} .
\end{aligned}
$$

Thus, $\tilde{\Sigma}_{m+3}=\alpha_{m-3} \beta_{m-4}(53)$

So that, $\tilde{k}_{m+3}=\frac{\alpha_{m-3} \beta_{m-4}^{2}}{\beta_{m-3}^{2}+\alpha_{m-3}^{2} \beta_{m-4}^{2}}(54)$

Then, $\tilde{\theta}_{t j \mid t}=\tilde{\theta}_{t_{j \mid t-1}}+\tilde{k}_{t}\left(y_{t}-A_{t-1} \hat{\theta}_{t}\right), t>t_{j}$ (55)

But $A_{t}=1$ at $t_{j}=m+2$ and $t=m+3$ so that (55) becomes

$$
\tilde{\theta}_{m+2 \mid m+3}=\tilde{\theta}_{m+2 \mid m+2}+\tilde{k}_{m+3}\left(y_{m+3}-\hat{\theta}_{m+3}\right)(56)
$$

Thus, $\hat{\theta}_{m+2 \mid m+2}=E\left[\theta_{m+2} \mid F_{m+2}^{y}\right]=E\left[\left(\alpha_{m-4} \theta_{m+1}+\right.\right.$ $\beta m-4 u m+2|F m+2 y=\alpha m-4 E \theta m+1| F m+2 y=\alpha m-4 \theta$ $m$ since we have made an observation up to time $t=m+$ 1 we have $\hat{\theta}_{m+1}=\hat{X}_{m+1}$ so that, $\hat{\theta}_{m+2 \mid m+2}=\alpha_{m-4} \hat{X}_{m+1}$.

Next, $\hat{\theta}_{m+3}=E\left[\theta_{m+3} \mid F_{m+2}^{y}\right]=E\left[\left(\alpha_{m-3} \theta_{m+2}+\right.\right.$ $\beta m-2 u m+3|F m+2 y=\alpha m-3 E \theta m+3| F m+2 y=\alpha m-3 \theta$ $m$ 开Rat is, $\hat{\theta}_{m+3}=\alpha_{m-3} \hat{\theta}_{m+2}$ and $\hat{\theta}_{m+2}=\alpha_{m-4} \hat{\theta}_{m+1}$ combines to give

$$
\hat{\theta}_{m+3}=\alpha_{m-3}\left(\alpha_{m-4} \hat{\theta}_{m+1}\right)=\alpha_{m-3} \alpha_{m-4} \hat{X}_{m+2} .
$$

Hence (56) gives $\hat{X}_{m+2 \mid m+3}=\alpha_{m-4} \hat{X}_{m+1}+$ $\tilde{k}_{m+3}\left(X_{m+3}-\alpha_{m-3} \alpha_{m-4} \hat{X}_{m+1}\right)$.

Substituting for $\tilde{k}_{m+3}$ we

have $\tilde{X}_{m+2 \mid m+3}=\alpha_{m-4} \hat{X}_{m+1}+\frac{\alpha_{m-3} \beta_{m-4}^{2}}{\beta_{m-3}^{2}+\alpha_{m-3}^{2} \beta_{m-4}^{2}}\left(X_{m+3}-\right.$ $a m-3 a m-4 X m+1$

This simplifies to give $\tilde{X}_{m+2 \mid m+3}=\frac{\alpha_{m-4}\left(\beta_{m-3}^{2}\right) \hat{X}_{m+1}+\alpha_{m-3} \beta_{m-4}^{2} X_{m+3}}{\beta_{m-3}^{2}+\alpha_{m-3}^{2} \beta_{m-4}^{2}}(57)$

We now list these models in order, to obtain the general pattern of obtaining estimate of missing observation at any stage. Hence we have;

1. $\tilde{X}_{m \mid m+1}=\frac{\alpha_{m-2}\left(\beta_{m-1}^{2}\right) X_{m-1}+\alpha_{m-1} \beta_{m-2}^{2} X_{m+1}}{\beta_{m-1}^{2}+\alpha_{m-1}^{2} \beta_{m-2}^{2}}$

$$
\text { 2. } \tilde{X}_{m \mid m+2}=
$$
$\frac{\alpha_{m-3}\left(\beta_{m-1}^{2} \alpha_{m-1}^{2} \beta_{m-2}^{2}\right) X_{m-1}+\alpha_{m-1} \alpha_{m-2}^{2} \beta_{m-3}^{2} X_{m+2}}{\beta_{m-1}^{2}+\alpha_{m-1}^{2} \beta_{m-2}^{2}+\alpha_{m-1}^{2} \alpha_{m-2}^{2} \beta_{m-3}^{2}}$

$$
\tilde{X}_{m \mid m+2}=\frac{\alpha_{m-3}\left(\beta_{m-2}^{2}\right) \hat{X}_{m}+\alpha_{m-2} \beta_{m-3}^{2} X_{m+2}}{\beta_{m-2}^{2}+\alpha_{m-2}^{2} \beta_{m-3}^{2}}
$$

3. $\tilde{X}_{m \mid m+3}=$

$\frac{\alpha_{m-4}\left(\beta_{m-1}^{2} \alpha_{m-1}^{2} \beta_{m-2}^{2}+\alpha_{m-1}^{2} \alpha_{m-2}^{2} \beta_{m-3}^{2}\right) X_{m-1}+\alpha_{m-1} \alpha_{m-2} \alpha_{m-3} \beta_{n}^{2}}{\beta_{m-1}^{2}}$ $\beta_{m-1}^{2}+\alpha_{m-1}^{2} \beta_{m-2}^{2}+\alpha_{m-1}^{2} \alpha_{m-2}^{2} \beta_{m-3}^{2}+\alpha_{m-1}^{2} \alpha_{m-2}^{2} \alpha_{m-3}^{2} \beta_{m-4}^{2} d$

$$
\begin{gathered}
\tilde{X}_{m+1 \mid m+3} \\
=\frac{\alpha_{m-4}\left(\beta_{m-2}^{2} \alpha_{m-2}^{2} \beta_{m-3}^{2}\right) \hat{X}_{m}+\alpha_{m-2} \alpha_{m-3} \beta_{m-4}^{2} X_{m+3}}{\beta_{m-2}^{2}+\alpha_{m-2}^{2} \beta_{m-3}^{2}+\alpha_{m-2}^{2} \alpha_{m-3}^{2} \beta_{m-4}^{2}} \\
\tilde{X}_{m+2 \mid m+3}=\frac{\alpha_{m-4}\left(\beta_{m-3}^{2}\right) \hat{X}_{m+1}+\alpha_{m-3} \beta_{m-4}^{2} X_{m+3}}{\beta_{m-3}^{2}+\alpha_{m-3}^{2} \beta_{m-4}^{2}}
\end{gathered}
$$

$$
\begin{gathered}
\mathrm{S} . \tilde{X}_{m \mid m+s}= \\
\frac{\left[\begin{array}{c}
\alpha_{m-(s+1)}\left(\beta_{m-1}^{2}+\alpha_{m-1}^{2} \beta_{m-2}^{2}+\alpha_{m-1}^{2} \alpha_{m-2}^{2} \beta_{m-3}^{2}+\cdots+\alpha_{m-1}^{2} \alpha_{m-2}^{2} \ldots \alpha_{m-(s+1)}^{2} \beta_{m-s}^{2}\right) X \\
\alpha_{m-1} \alpha_{m-2} \ldots \alpha_{m-s} \beta_{m-(s+1) X}^{2} X_{m+s}
\end{array}\right.}{\beta_{m-1}^{2}+\alpha_{m-1}^{2} \beta_{m-2}^{2}+\alpha_{m-1}^{2} \alpha_{m-2}^{2} \beta_{m-3}^{2}+\cdots+\alpha_{m-1}^{2} \alpha_{m-2}^{2} \ldots \alpha_{m-s}^{2} \beta_{m-(s+1)}^{2}} \\
\tilde{X}_{m+(s-1) \mid m+s} \\
=\frac{\alpha_{m-(s+1)}\left(\beta_{m-s}^{2}\right) \hat{X}_{m+(s-2)}+\alpha_{m-s} \beta_{m-(s+1)}^{2} X_{m+s}}{\beta_{m-s}^{2}+\alpha_{m-s}^{2} \beta_{m-(s+1)}^{2}}
\end{gathered}
$$

The estimate of $\tilde{X}_{m+1 \mid m+2}$ are the same as those obtained by [2] and [19]. The approach in section 3 can only handle some of the nonlinear models mentioned earlier. Let's give an example of AR (1) model with a constant conditional variance, $\beta_{t}^{2}=\sigma^{2}$ and $\alpha_{t}=\phi$ (See [1].

In this case the estimates of the missing observations $X_{m}, X_{m+1}, X_{m+2}, X_{m+3}$ for an AR(1) model from 1 up to $S$ missing values will be as follows;

$$
\text { 1. } \tilde{X}_{m \mid m+1}=\frac{\phi X_{m-1}+\phi X_{m+1}}{1+\phi^{2}}
$$

2. $\tilde{X}_{m \mid m+2}=\frac{\phi\left(1+\phi^{2}\right) X_{m-1}+\phi^{2} X_{m+2}}{1+\phi^{2}+\phi^{4}}$

$$
\tilde{X}_{m \mid m+2}=\frac{\phi \hat{X}_{m}+\phi X_{m+2}}{1+\phi^{2}}
$$

3. $\tilde{X}_{m+2 \mid m+3}=\frac{\phi\left(1+\phi^{2}+\phi^{4}\right) X_{m-1}+\phi^{3} X_{m+3}}{1+\phi^{2}+\phi^{4}+\phi^{6}}$

$$
\begin{gathered}
\tilde{X}_{m+1 \mid m+3}=\frac{\phi\left(1+\phi^{2}\right) \hat{X}_{m}+\phi^{2} X_{m+3}}{1+\phi^{2}+\phi^{4}} \\
\tilde{X}_{m+2 \mid m+3}=\frac{\emptyset \hat{X}_{m+1}+\emptyset X_{m+3}}{1+\phi^{2}} \\
\text { S. } \tilde{X}_{m \mid m+s}=\frac{\emptyset\left(1+\phi^{2}+\phi^{4}+\cdots+\phi^{2(s-1)} X_{m+s}\right)+\phi^{s} X_{m+s}}{1+\phi^{2}+\phi^{4}+\cdots+\phi^{2 s}} \\
\tilde{X}_{m+(s-1) \mid m+s}=\frac{\emptyset X_{m+(s-2)}+\emptyset X_{m+s}}{1+\phi^{2}}
\end{gathered}
$$

Hence the general form of these sequences of estimates is given by;

$$
\tilde{X}_{m+j \mid m+i}=\frac{\emptyset\left(\sum_{\mathrm{r}=0}^{\mathrm{i}-\mathrm{j}-1} \phi^{2 \mathrm{r}}\right) \mathrm{X}_{\mathrm{m}+\mathrm{j}-1}+\phi^{\mathrm{i}-\mathrm{j}} \mathrm{X}_{\mathrm{m}+\mathrm{i}}}{\sum_{\mathrm{r}=0}^{\mathrm{i}-\mathrm{j}} \phi^{2 \mathrm{r}}}(58)
$$

Where $j=0,1,2, \ldots, s-1$ and $i=1,2,3, \ldots, s$ for $i>j$.

\section{An empirical study}

In this section empirical study is carried out to illustrate the results obtained in section three on simulated AR (1) data. Some values are withheld and then estimated as though they were missing.

Simulated AR (1) data and corresponding estimated missing values generated using formula (77) in section three are compared.

Tables 1 and 2 and tables 4.1 and 4.2 gives actual data and estimates of missing values for different values of the parameter $\phi$ and initial value $X_{0}$.

Estimation of missing observations on simulated AR 


\section{(1) data.}

AR (1) process is written as $X_{t}=\phi X_{t-1}+e_{t}$, where $e_{t}$ is a purely random process which is normally distributed with mean zero and unit variance (i.e. $e_{t} \sim N(0,1), \phi$ is some constant given within the range $|\phi|<1$ and $X_{0}$ is the initial observation which we choose to determine the size of the data.

Hence we have,

$$
\left.\begin{array}{c}
X_{1}=\phi X_{0}+e_{1} \\
X_{2}=\phi X_{1}+e_{2} \\
\vdots \\
X_{n}=\phi X_{n-1}+e_{n}
\end{array}\right\}
$$

We generated AR (1) data using a computer programme. The general formula (58) in section three was then applied to the simulated AR (1) data with several consecutive missing observations which were artificially created. Table 1.1 illustrates the missing values and their estimates and Tables 1.2 illustrate the same for different values of $\phi$ and $X_{0}$.

If we set $\phi=0.86$ and $X_{0}=100$, we obtain the following $\operatorname{AR}(1)$ data for the first 20 values.

Table 1. Eighteen consecutive missing values from position 2 to 19 with their estimates.

\begin{tabular}{|c|c|c|c|}
\hline$t$ & $\begin{array}{l}\text { Actual AR(1) } \\
\text { data }\left(X_{t}\right)\end{array}$ & $\begin{array}{l}\text { Data with missing } \\
\text { values }\end{array}$ & Estimated values \\
\hline 1 & 86.21399 & 86.21399 & \\
\hline 2 & 73.03785 & - & 74.11012 \\
\hline 3 & 63.71109 & - & 63.69526 \\
\hline 4 & 53.52442 & - & 54.73207 \\
\hline 5 & 47.14499 & - & 47.01625 \\
\hline 6 & 38.94666 & - & 40.37197 \\
\hline 7 & 35.22709 & - & 34.6478 \\
\hline 8 & 31.41615 & - & 29.71327 \\
\hline 9 & 25.95318 & - & 25.45593 \\
\hline 10 & 21.70792 & - & 21.77875 \\
\hline 11 & 17.5829 & - & 18.59792 \\
\hline 12 & 15.35208 & - & 15.84095 \\
\hline 13 & 12.63766 & - & 13.44501 \\
\hline 14 & 11.30084 & - & 11.35549 \\
\hline 15 & 9.111297 & - & 9.524767 \\
\hline 16 & 8.188405 & - & 7.91112 \\
\hline 17 & 6.679135 & - & 6.477774 \\
\hline 18 & 5.224529 & - & 5.192006 \\
\hline 19 & 3.283825 & - & 4.024678 \\
\hline 20 & 2.94902 & 2.94902 & \\
\hline
\end{tabular}

Positions of missing values Calculated $\chi^{2}$ value Table value $\chi_{0.05,4}^{2}$

\begin{tabular}{lll}
\hline $2-19$ & 0.53467 & 27.59 \\
\hline
\end{tabular}

We generate other 20 different values of AR (1) with $\phi=0.5$ and $X_{0}=1000000$ as illustrated in Table 1.2.
Table 2. Eighteen consecutive missing values from position 2 to 19 with their estimates.

\begin{tabular}{llll}
\hline & $\begin{array}{l}\text { Actual AR(1) } \\
\mathbf{t a}\left(X_{t}\right)\end{array}$ & $\begin{array}{l}\text { Data with missing } \\
\text { values }\end{array}$ & Estimated values \\
\hline 1 & 500000 & 500000 & 250000 \\
2 & 249999.8 & - & 125000 \\
3 & 125001.2 & - & 62500 \\
4 & 62500.23 & - & 31250 \\
5 & 31250.01 & - & 15625 \\
6 & 15622.97 & - & 7812.5 \\
7 & 7809.437 & - & 3906.25 \\
8 & 3905.664 & - & 1953.125 \\
9 & 1953.414 & - & 976.5632 \\
10 & 977.1117 & - & 488.2828 \\
11 & 488.8349 & - & 244.1437 \\
12 & 244.358 & - & 122.0765 \\
13 & 122.5056 & - & 61.04752 \\
14 & 61.77014 & - & 30.54231 \\
15 & 33.05295 & - & 15.30825 \\
16 & 15.78969 & - & 7.728314 \\
17 & 7.215367 & - & 4.012536 \\
18 & 3.808213 & - & 2.303026 \\
19 & 2.473077 & - & \\
20 & 1.745028 & 1.745028 & \\
\hline & & & Table value $\chi^{2} .05,17$ \\
\hline Positions of missing & Calculated $\chi^{2}$ value & \\
values & & & 27.59 \\
& $2-19$ & 0.27716 & \\
\hline & & & - \\
\hline
\end{tabular}

Chi-square goodness of fit statistics was calculated to compare the estimated and actual values. Each of the chisquare values were not significant for any reasonable level of significance which confirms that the estimated values agree well with the actual values.

\section{Conclusions}

Most methods developed for estimation of missing observations in time series Analysis, have been limited to the case of one or two consecutive missing observations. In this paper, we have employed the state space models which can handle irregularly spaced data. Missing observations in a Time Series can safely be treated as special case of such data. In particular, we have extended the formula derived in [1] to encompass the case where there are several consecutive missing observations. In a special case, we apply the formula on AR (1) model and it performs satisfactorily, since it has only one parameter.

\section{References}

[1] Abraham B. and Thaveneswaran, A. (1991). A nonlinear 
Time Series model and estimation of missing observation. Ann. Inst. Stat.Math Vol 43, $422-528$.

[2] Abraham B.(1981) Missing observation in time series, Common Statistics A- Theory Methods, 10, 1645-1653.

[3] Ansley, C.F and Kohn, R. (1883). Exact likelihood of vector autoregressive-moving average process with missing aggregated data. Biometrika 70, 275-278.

[4] Jones, R.H (1985). Time series analysis with unequally space data. In:E.J. Hannan, P. R.Krishnaiah and M. M. Rao, Eds., Handbook of statistics, vol.5. North-Holland, Amsterdam, 157-177.

[5] Penza, J. and Shea, B. (1997). The exact likelihood of an autoregressive-moving average model with incomplete data. Biometrika 84, 919-928.

[6] Nassiuma. D. (1994). SymmetricStable Sequences with missing observations. J. Time Ser. Anal. 15, 313-323.

[7] Palma, W. and Chan, N. H. (1997). Estimation and forecasting of long-memory processes. J. Forecasting 16, 395-410.

[8] Chan,N. H and Palma, W. (1998). State space modeling of long memory process. Ann statist. 26, 719-740.

[9] Palma, W. and Del Pino, G. (1999). Statistical analysis of incomplete long-range dependent data. Biometrika, 86, 4, 965-972.

[10] Engle, R.F. (1982). Autoregressive conditional heteroscedasticity with estimates of the variance of United Kingdom inflation, Econometrica, 50, 987-1007.

[11] Harrison, P.J. and stevens, C.F. (1976). Bayesian forecasting (with discussion), J. Roy. Statist. Soc.Ser. B, 38, 205-248.

[12] Tjostheim, D (1986). Estimation in nonlinear time series models, stochastic process. Appl. 21, 251-273.

[13] Thavaneswaran, A and Abraham, B. (1988). Estimation for nonlinear timeseries models using estimating equations. J. Time Ser. Anal. 9, 99-108.

[14] Nichols, D.F and B.G. Quinn (1982). Random coefficient Autoregressivemodels: An introduction lecture notes in statistics no. 11. Springer-Verlag, New York.

[15] Ozaki, T. (1985). Nonlinear time series models and dynamic systems, handbook of statistics, vol. 5 (eds, E.J. Hannan, P.R Krishnaiah and M.M. Rao), 25-83, North Holland, Amsterdam.

[16] Brockwel, P. J. and Davis, R. A. (1987). Time series: Theory and Methods, Springer, New York.

[17] Shiryayev, A.N. (1984). Probability, Graduate test in Math., 95, Springer, New York.

[18] Charbonnier, R., Barlaud, M., Alengrin, G. and Menez, J. (1987). Results on AR- modeling of nonstationary signals, Signal Process., 12, 143-1587.

[19] Miller, R. B. and Ferreiro, O. (1984). A strategy to complete a time series with missing observations, Lecture Notes in statistics, 25, 251-275, Springer, New York. 